\title{
EVALUACIÓN DEL PESO DEL LEÑO A PARTIR DE VARIABLES DIMENSIONALES EN DOS ESPECIES DE MEZQUITE PROSOPIS ARTICULATA S. WATSON Y P. PALMERI S. WATSON, EN BAJA CALIFORNIA SUR, MÉXICO
}

\author{
José Luis León de la Luz, Raymundo Domínguez Cadena y \\ Sara Cecilia Díaz Castro
}
Programa de Planeación Ambiental, Centro de Investigaciones Biológicas del Noroeste (CIBNOR). Apdo. postal 128, 23000 La Paz, Baja California Sur, México correo electrónico: jlleon04@cibnor.mx

\section{RESUMEN}

El presente trabajo se propone definir, mediante modelos de regresión, un procedimiento sencillo de evaluación de la biomasa fresca del leño de dos especies de mezquite (Prosopis articulata y P. palmeri), actualmente aprovechadas como materia prima para elaborar carbón vegetal en la Sierra de La Giganta, Baja California Sur, México. Esta práctica se ha realizado desde hace varias décadas, pero sólo desde 1990 se cuenta con estadísticas basadas en permisos otorgados. Las variables independientes que mejor se correlacionaron con el peso fresco del leño en una muestra destructiva fueron el perímetro basal del tronco en el caso de $P$. articulata $\left(R^{2}=0.98, F=394.12\right)$, y la cobertura del dosel en $P$. palmeri $\left(R^{2}=0.94, F=63.26\right)$, los modelos empíricos empleados fueron el logarítmico y el exponencial, respectivamente. Para aplicar las fórmulas encontradas se procedió a determinar en muestreos no destructivos en 11 superficies de una hectárea el valor de la variable independiente respectiva en 1,283 individuos de P. palmeri y 755 de P. articulata. De esta manera se calculó que el peso de leño en pie útil para la elaboración de carbón para $P$. articulata es de $37,040 \mathrm{~kg} / \mathrm{ha}$ y para $P$. palmeri es de $2,045 \mathrm{~kg} / \mathrm{ha}$. De acuerdo con las tasas de extracción autorizadas oficialmente durante la década de 1990, la biomasa del leño calculada correspondería a un aprovechamiento anual de todos los individuos de tallas adecuadas para el propósito (diámetro basal del tronco $>10 \mathrm{~cm}$ ) de $P$. articulata en 179 ha en los cauces de arroyos, o bien de 3,928 ha en mesas y lomeríos en el caso de que se hubieran explotado exclusivamente individuos de P. palmeri.

Palabras clave: análisis dimensional, Baja California Sur, carbón, mezquite, Prosopis.

\begin{abstract}
Using regression models, this research determines the fresh woody biomass for two species of mezquite (Prosopis articulata and P. palmeri) currently used as raw material to
\end{abstract}


make charcoal in Sierra de la Giganta, Baja California Sur, Mexico. This practice began many decades ago, but only since 1990 production data based on issued permissions are available. The independent variables better correlated with the fresh wood weight in a destructive sample were the basal perimeter of the stems for $P$. articulata $\left(R^{2}=0.98\right.$, $F=394.12)$, and the canopy coverage for $P$. palmeri $\left(R^{2}=0.94, F=63.26\right)$. The empirical models employed in these calculations were logarithmic and exponential, respectively. In order to apply these formulas, the appropriate independent variable for each species was used to estimate fresh wood weight in 11 plots of one hectare. 1,283 individuals of $P$. palmeri and 755 individuals of $P$. articulata were non-destructively sampled. By employing these calculations, we determined that the biomass useful for charcoal production was $37,040 \mathrm{~kg}$ / ha for $P$. articulata and $2,045 \mathrm{~kg} / \mathrm{ha}$ for $P$. palmeri. If each species is harvested exclusively and in accordance with the quantity of wood officially authorized to be gathered during the decade of 1990, these values represent an annual harvest for all the individuals with the adequate size for this purpose (trunk basal diameter $>10 \mathrm{~cm}$ ) of 179 hectares of $P$. articulata along riparian areas or 3,928 hectares of $P$. palmeri on mesas and slopes.

Key words: Baja California Sur, charcoal, dimensional analysis, mezquite, Prosopis.

\section{INTRODUCCIÓN}

Los mezquites son especies vegetales arbóreas y arbustivas termo-xerófilas. Son componentes fisonómicamente importantes en la vegetación de las zonas áridas y semi-áridas del planeta, particularmente en el continente americano. En muchas áreas, los individuos de mezquite constituyen las plantas de mayor talla y longevidad, y ofrecen una gama de recursos aprovechados por el gremio de consumidores: néctar, polen, frutos, semillas y follaje. Además, su dosel es un refugio para la fauna de vertebrados, y es reconocido como parte importante en la dinámica de la vegetación en esas zonas a través del proceso de nodricismo (Vandermeer, 1980; Carrillo-García et al., 1999).

El mezquite es también un importante recurso para los habitantes del medio rural, pues el leño de troncos y ramas representan una fuente de energía calorífica de primera calidad. Un aspecto refinado de este uso es la elaboración de carbón, actividad que implica cierto valor agregado y que puede ser practicada de manera sustentable.

En la Sierra de La Giganta de Baja California Sur (México) se encuentran dos especies de mezquite. El más común es el "mezquite amargo", Prosopis articulata, que habita preferentemente a lo largo de cauces de arroyos temporales y secundariamente en planicies aluviales, desde el nivel del mar hasta unos 350 $\mathrm{m}$ de elevación. La otra especie, P. palmeri, es localmente conocida como "palo fierro", debido a la dureza de su leño y se encuentra restringida a esta serranía, 
ubicándose en laderas y mesetas rocosas desde $100 \mathrm{~m}$ hasta $500 \mathrm{~m}$ de altitud (Turner et al., 1995).

Además de su valor ecológico, estas dos especies han constituido las principales fuentes de combustible para uso humano y antaño lo fueron para la construcción rural. En las últimas décadas se ha intensificado el proceso de elaboración de carbón a partir del leño de estas dos especies, la producción es vendida al equivalente de $\$ 0.15$ dólares el kilo (2003) en la capital estatal (La Paz) y eventualmente es exportada a los Estados Unidos. A través de intermediarios el precio se incrementa sustancialmente.

Cifras oficiales (Anónimo, 1998) revelan un promedio anual de 8 a 9 autorizaciones dentro del período 1990-1997 para la elaboración de carbón a partir de estas especies, basadas en la extracción de $34,068.14 \mathrm{~m}^{3}$ de leño en rollo en el municipio de Comondú y seis autorizaciones para extraer $15,838.5 \mathrm{~m}^{3}$ en el municipio de La Paz. Estos números podrían ser inferiores a las cantidades realmente aprovechadas, debido al clandestinaje. El volumen anotado se refiere al leño sin distinción de especies, pero correspondería principalmente al tronco y ramas de "mezquite amargo" con diámetros superiores a $10 \mathrm{~cm}$, ya que estas dimensiones son las mínimas útiles para el propósito de producción de carbón. Así también se estima que una tonelada de carbón se produce a partir de $5 \mathrm{~m}^{3}$ de leño en rollo, sin distinción de la especie (Osuna Leal, 1989).

No es fácil evaluar el impacto ecológico por la extracción del leño, que sin duda existe, situación que podría manifestarse como una contingencia ambiental en el futuro de no tomarse las medidas adecuadas desde el presente, ya que la extracción repercute en la tasa de regeneración natural de las mismas especies y de aquellas otras que podrían requerir de su resguardo. De hecho, los habitantes de la zona de estudio declaran que el "palo fierro" ha dejado de ser ya la materia prima preferencial por la escasez de tallas adecuadas, y que ha sido sustituido por el "mezquite amargo".

Cabe señalar que las autoridades respectivas han pretendido llevar un control de la extracción, pero existen limitaciones, primero porque falta una vigilancia estrecha para evitar que se rebasen los límites establecidos, pero principalmente por la carencia absoluta de manejo silvícola, que debería basarse en la evaluación del recurso, así como en el conocimiento de la dinámica poblacional de cada especie y de su tasa de crecimiento.

El objetivo de esta investigación es el de estimar a nivel de poblaciones la disponibilidad del recurso para cada una de las especies de mezquite consideradas, específicamente el peso del leño en pie usado para la elaboración de carbón. La estimación se basa en la obtención de un modelo confiable, conformado con variables que impliquen mediciones no destructivas de los individuos y de rápida obtención. Las variables se integran en modelos predictivos representados por la 
mejor regresión estadística definida empíricamente. La intención última es aportar información para que las autoridades estatales y federales puedan usarla para planificar las tasas de extracción de este recurso, mismas que también deberán acompañarse de medidas de mitigación. El presente estudio forma parte de otro más amplio que pretende establecer las bases de un manejo sustentable de las especies para proteger el recurso y mejorar las expectativas de vida de la población rural que habita en las rancherías de la Sierra de la Giganta.

\section{MATERIALES Y MÉTODOS}

Área de estudio

El área de estudio se localiza al este de Ciudad Constitución, Baja California Sur. Corresponde a la vertiente occidental de la Sierra de la Giganta, la cual fisiográficamente se caracteriza por estar esculpida por arroyos intermitentes que en ocasiones conducen grandes volúmenes de agua hacia el área de Bahía Magdalena, en el Océano Pacífico, atravesando llanuras de pendiente suave a lo largo de hasta $100 \mathrm{~km}$ de distancia. Fitogeográficamente, se encuentra en la provincia del Desierto Sonorense, dentro de la subprovincia de las llanuras de (Bahía) Magdalena (Shreve y Wiggins, 1964; Wiggins, 1980). Genéricamente, su vegetación se ubica en la categoría de matorral xerófilo (Rzedowski, 1978). En algunos recodos de arroyos se han logrado formar valles aluviales en los cuales prevalecen suelos profundos de textura fina, en donde, si existe agua disponible, se practican de manera limitada actividades agrícolas de subsistencia.

La asociación vegetal de los márgenes de los arroyos corresponde a la denominada como mezquital (Rzedowski, 1978; Anónimo, 1981a), misma que se encuentra alterada por las actividades humanas y el ganado doméstico; las especies que la caracterizan son Prosopis articulata, Schoepfia californica, Vallesia glabra y Acacia farnesiana. En las mesas y lomeríos la cubierta vegetal corresponde al matorral sarcocaule (Anónimo, 1981a); los dominantes fisonómicos son Jatropha cuneata, Prosopis palmeri, Opuntia cholla, Fouquieria diguetii, Bursera microphylla, Pachycereus pringlei y Larrea tridentata. Aquí el suelo se caracteriza por presentar altos niveles de pedregosidad, superior a $90 \%$ en la superficie.

El clima corresponde a la fórmula $\mathrm{BW}\left(\mathrm{h}^{\prime}\right) \mathrm{hw}\left(\mathrm{x}^{\prime}\right)$, es decir, muy cálido, muy seco, con régimen de lluvias de verano, así como con escasa precipitación invernal; la estación meteorológica más cercana (Cd. Constitución) señala una acumulación promedio anual de $149 \mathrm{~mm}$ de lluvia y una temperatura media anual de $22{ }^{\circ} \mathrm{C}$, con base en un registro de 18 años (Anónimo, 1981b). 
Metodología aplicada

El sitio elegido para la realización del estudio corresponde al arroyo La Picota-Tepentú (Fig. 1). Los muestreos destructivos se realizaron en las inmediaciones de la ranchería de Tepentú $\left(25^{\circ} 08^{\prime} \mathrm{N}\right.$ y $111^{\circ} 25^{\prime} \mathrm{W}$, a $90 \mathrm{~m}$ s.n.m. $)$ durante el verano de 1999. Se eligieron seis individuos de $P$. palmeri y seis de $P$. articulata, en los que se determinaron las siguientes cuatro medidas dasométricas: a) altura, b) cobertura (considerando la proyección de la copa como una elipse, se midió un diámetro largo y otro corto), c) altura a la primera ramificación y d) perímetro basal del tronco. La elección de los individuos se realizó a priori tratando de abarcar la diversidad de dimensiones en la población para cada especie vegetal, partiendo de los tamaños mínimos propios para la explotación (diámetro basal superior a $10 \mathrm{~cm}$ ). Se derribó cada uno de estos árboles y se pesó el leño útil para fabricación de carbón (con diámetro de ramas y tallos mayor de $10 \mathrm{~cm}$ ). El permiso respectivo para el derribo fue otorgado bajo el número 03/388/98 por la delegación en Baja California Sur de la Secretaría del Medio Ambiente y de Recursos Naturales.

Los datos de cada especie se sometieron para su análisis de regresión con ayuda del programa Statistica 5.0, usando como variable dependiente (y) el peso del leño y como variables independientes $(x)$ el resto de las medidas dasométricas. La relación entre las variables se analizó por medio de las siguientes siete opciones de correlación:

(1) lineal simple: $y=b x+a$

(2) exponencial: $y=\mathrm{e}^{(\mathrm{a}+\mathrm{b} x)} \quad \mathrm{o} \quad \ln (y)=\mathrm{b} x+\mathrm{a}$

(3) logarítmica: $y=a x^{b} \quad$ o $\quad \ln (y)=b \ln (x)+\ln (a)$

(4) semi-logarítmica-x: $y=\mathrm{b} \ln (x)+\mathrm{a}$

(5) recíproca: $1 / y=\mathrm{b} x+\mathrm{a}$ o $y=1 /(\mathrm{b} x+\mathrm{a})$

(6) cuadrática-x: $y=b x^{2}+a$

(7) de raíz cuadrada-y: $\sqrt{ } y=b x+a$

Siguiendo el criterio de varios autores, como Murray y Jacobson (1982), Vora (1988) y Patón et al. (1998), se usó el coeficiente de determinación $R^{2}$ (el cuadrado del coeficiente de correlación simple $r$ ) como el criterio para obtener el mejor modelo de regresión. Este estimador cuantifica la relación de la suma de cuadrados de las desviaciones de los datos individuales con respecto a los correspondientes en el modelo obtenido (diferencia que da lugar a la suma de cuadrados de los residuales o del error); en otras palabras, representa la proporción de la variación de los datos explicada por el modelo respectivo. El valor 1 (o 100\%) supone el ajuste perfecto de los puntos sobre el modelo (Zar, 1984). Todos los modelos de 
$111^{\circ} 25^{\prime} \mathrm{W}$

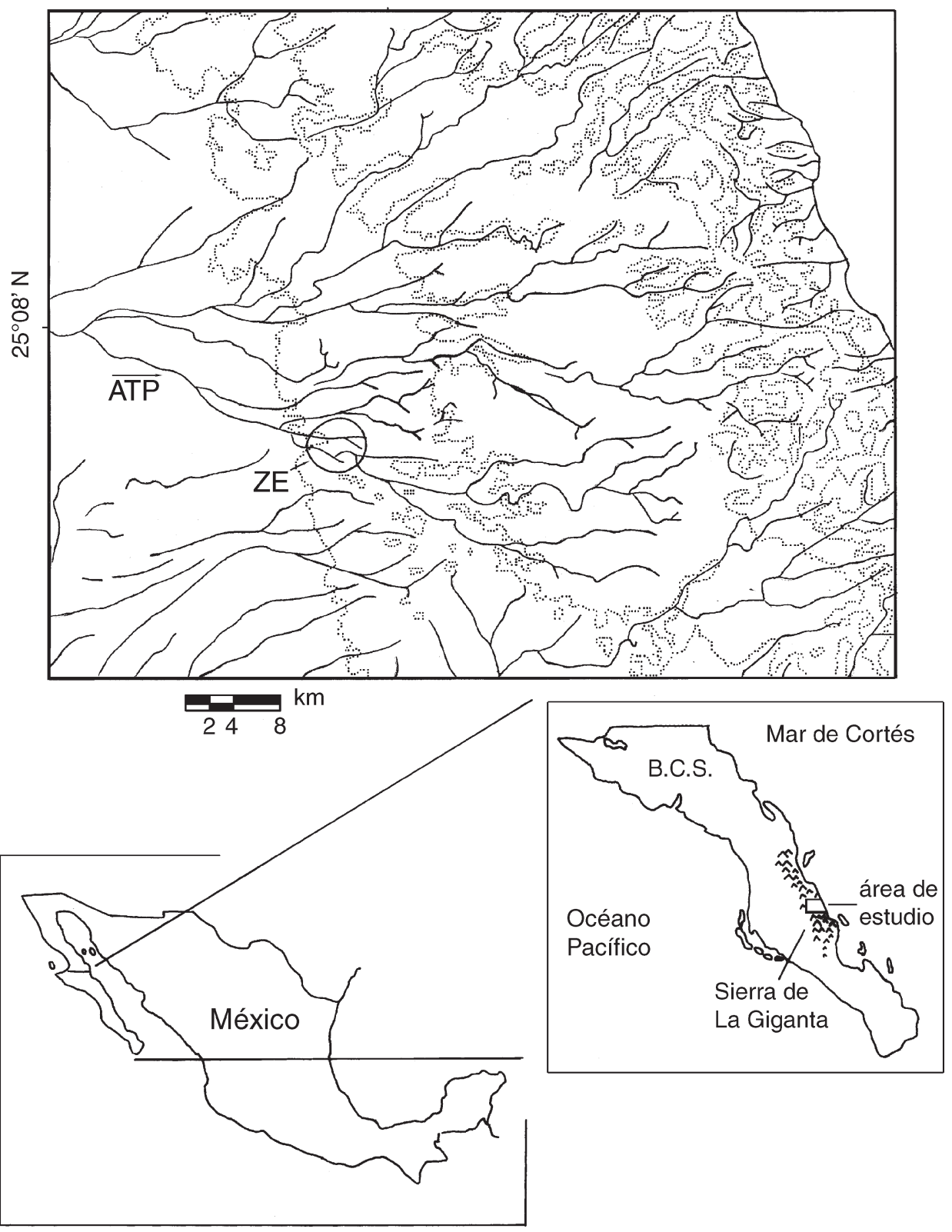

Fig. 1. Ubicación geográfica de la Sierra de La Giganta en Baja California Sur, México. Zona de estudio (ZE) en el arroyo Tepentú-La Picota (ATP), donde se realizaron los muestreos destructivos y no destructivos. La superficie de la cuenca del arroyo es de $723 \mathrm{~km}^{2}$ situados arriba de la cota de $100 \mathrm{~m}$ sobre el nivel del mar. 
regresión fueron probados mediante el cálculo de $F$ para comparar su varianza residual. A mejor ajuste de los puntos, es decir a mayor valor de $R^{2}$, se obtienen $F$ altamente significativas, $p<<0.05$ (Kvalseth, 1985).

Una vez obtenido y valorado el mejor modelo para cada especie, en una segunda etapa del estudio, se procedió a realizar mediciones en la misma área de trabajo en poblaciones de ambas especies de la variable que mejor se relacionó con el peso del leño. Para ello se realizaron cinco muestreos de 1 ha $(100 \times 100 \mathrm{~m})$ para $P$. palmeri y seis para $P$. articulata. Obtenidas las determinaciones de las variables para cada caso, se procedió a integrarlas en el modelo respectivo para calcular el peso del leño fresco en esa unidad de superficie.

Para contar con datos del peso específico del leño de cada especie de mezquite, se realizaron determinaciones del peso respectivo de un volumen conocido, tanto en condición de leño fresco (considerado como el punto de inflexión en la ganancia de peso al someterla a condiciones de humedad), como en la condición seca $\left(2 \mathrm{~h}\right.$ en un horno a $\left.45^{\circ} \mathrm{C}\right)$.

\section{RESULTADOS}

Producto del muestreo destructivo en seis ejemplares de cada especie, el Cuadro 1 señala los registros para las cuatro variables independientes consideradas para la obtención de los modelos empíricos (x, altura de la primera rama, perímetro basal, cobertura del dosel, y altura total del individuo) y la variable dependiente obtenida (y, peso fresco del individuo). El Cuadro 2 muestra los valores de los estimadores $R^{2}$ y $F$ obtenidos en cada caso para las dos especies de Prosopis con respecto a los pares de variables comparadas (x, y) en las siete opciones de correlación empleadas. En negritas se señalan los mejores ajustes de acuerdo con los mejores niveles de los estimadores.

Mientras que en P. palmeri se obtuvo la máxima correlación con el modelo exponencial (ecuación 2) en la relación cobertura-peso $\left(R^{2}=0.94 ; F=63.26\right)$, en $P$. articulata la misma se logró usando la opción logarítmica (ecuación 4) en la relación perímetro basal-peso del leño $\left(R^{2}=0.98 ; F=394.12\right)$. En estos modelos los valores de la pendiente (a) y la intercepción (b) son: para $P$. articulata a $=5.802$; $\mathrm{b}=2.618$, y para P. palmeri $\mathrm{a}=0.797 ; \mathrm{b}=0.177$.

Cabe observar que en el caso de $P$. articulata se obtuvieron coeficientes de $R^{2}$ de 0.98 para la correlación perímetro basal-peso en dos distintos modelos empíricos, pero el semi-logarítmico muestra una $F$ más significativa $(F=394.12$, $p<<0.05)$ que el de raíz cuadrática en $y(F=210.93)$. Un mayor valor en $F$ indica una menor varianza residual de los puntos o mejor ajuste entre los mismos; por tal razón, sólo el primer modelo se usó para esta especie en la fase de las estimaciones. 
Cuadro 1. Datos recabados de los muestreos destructivos de seis individuos de Prosopis palmeri (palo fierro) y otros tantos de Prosopis articulata (mezquite amargo) en el área del arroyo Tepetú-La Picota, municipio de Comondú, Baja California Sur (pie de la Sierra de La Giganta). El peso del leño fresco es la variable independiente $(y)$ en los modelos de regresión.

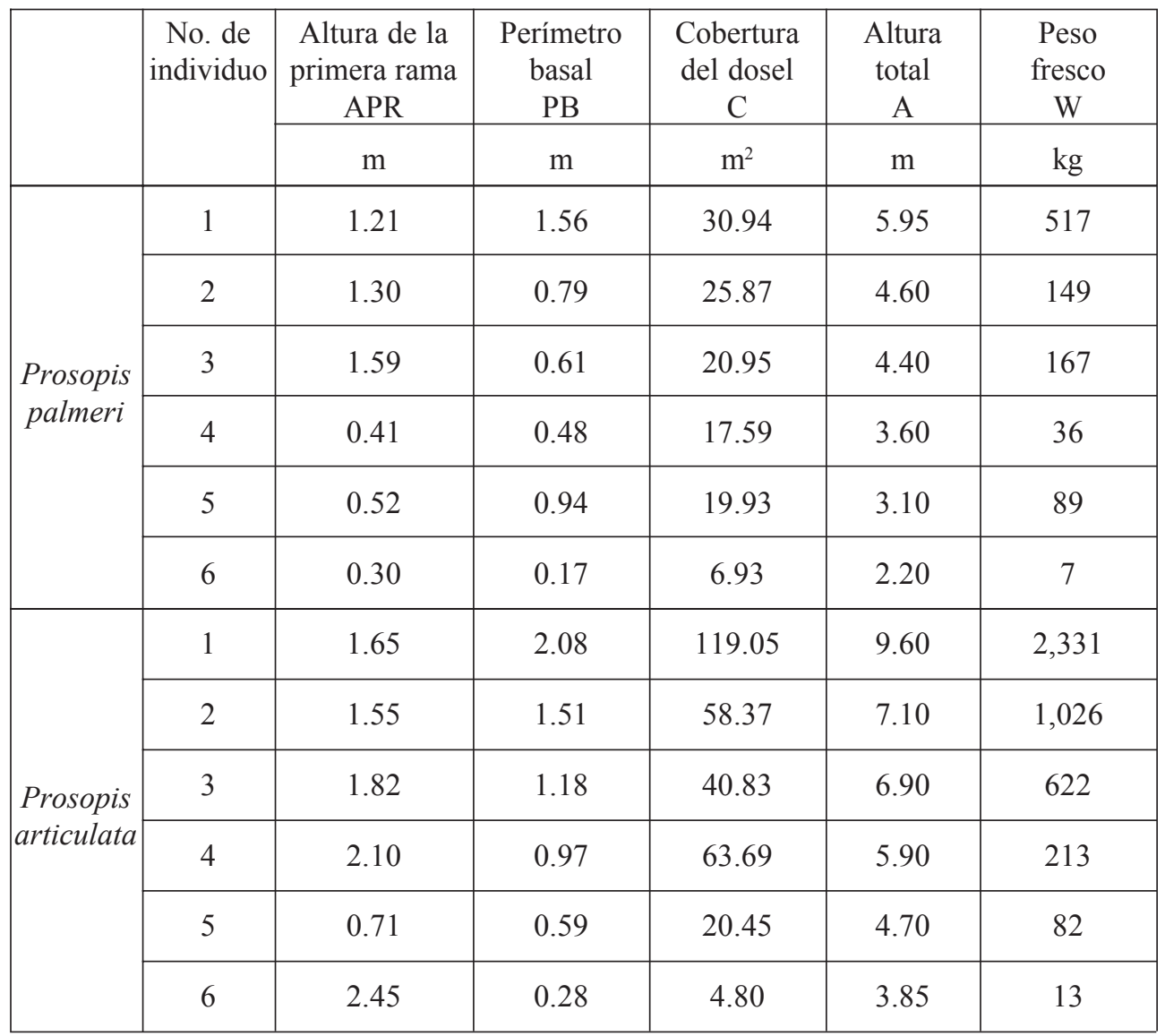

El Cuadro 3 muestra los resultados de la segunda etapa del estudio, consecuencia de la ejecución de muestreos no destructivos en superficies de 1 ha para cada especie de mezquite (cinco sitios para $P$. palmeri y seis para $P$. articulata), en las cuales sólo se realizaron mediciones de la variable independiente seleccionada para el caso (Cuadro 2). Una vez sustituidos esos valores en el modelo respectivo de cada especie, se obtiene la cantidad del peso fresco del leño por unidad de superficie. Se señalan asimismo el número de individuos muestreados en cada sitio, 
Cuadro 2. Coeficientes de determinación $R^{2}$ y su correspondiente estimación de varianza por el valor $F$ para los siete modelos empíricos de regresión evaluados. Variables independientes: $(x)$ APR: altura de la primera ramificación, PB: perímetro basal, C: cobertura del dosel o copa, A: altura del árbol. Variable dependiente $(y) \mathrm{W}$ : peso fresco del leño.

\begin{tabular}{|c|c|c|c|c|c|c|c|}
\hline & Modelo & & APR-W & PB-W & C-W & A-W & \multirow{15}{*}{$\begin{array}{c}\text { Prosopis } \\
\text { palmeri }\end{array}$} \\
\hline \multirow[t]{2}{*}{1} & Lineal & $R^{2}$ & 0.33 & 0.81 & 0.66 & 0.79 & \\
\hline & & $F^{2}$ & 2.05 & 17.83 & 7.98 & 15.97 & \\
\hline \multirow[t]{2}{*}{2} & Exponencial & $R^{2}$ & 0.61 & 0.76 & 0.94 & 0.84 & \\
\hline & & $F^{2}$ & 6.28 & 13.36 & 63.26 & 23.33 & \\
\hline \multirow[t]{2}{*}{3} & Logarítmico & $R^{2}$ & 0.74 & 0.89 & 0.91 & 0.88 & \\
\hline & & $F^{2}$ & 11.71 & 33.01 & 41.66 & 30.01 & \\
\hline \multirow[t]{2}{*}{4} & Semi-logarítmico & $R^{2}$ & 0.40 & 0.57 & 0.47 & 0.67 & \\
\hline & & $F^{2}$ & 2.72 & 5.37 & 3.55 & 8.27 & \\
\hline \multirow[t]{2}{*}{5} & $1 / x$ & $R^{2}$ & 0.42 & 0.32 & 0.31 & 0.54 & \\
\hline & & $F^{2}$ & 3.01 & 1.40 & 1.84 & 4.70 & \\
\hline \multirow[t]{2}{*}{6} & $x^{2}$ & $R^{2}$ & 0.24 & 0.89 & 0.81 & 0.89 & \\
\hline & & $F^{2}$ & 1.30 & 33.52 & 17.78 & 33.51 & \\
\hline \multirow[t]{2}{*}{7} & Raíz cuadrada $y$ & $R^{2}$ & 0.51 & 0.85 & 0.84 & 0.89 & \\
\hline & & $F^{2}$ & 4.23 & 23.67 & 21.31 & 34.25 & \\
\hline \multirow[t]{2}{*}{1} & Lineal & $R^{2}$ & 0.008 & 0.88 & 0.81 & 0.89 & \multirow{14}{*}{$\begin{array}{l}\text { Prosopis } \\
\text { articulata }\end{array}$} \\
\hline & & $F^{2}$ & 0.034 & 30.62 & 17.30 & 35.73 & \\
\hline \multirow[t]{2}{*}{2} & Exponencial & $R^{2}$ & 0.062 & 0.92 & 0.72 & 0.89 & \\
\hline & & $F^{2}$ & 0.264 & 47.32 & 10.53 & 34.26 & \\
\hline \multirow[t]{2}{*}{3} & Logarítmico & $R^{2}$ & 0.003 & 0.89 & 0.87 & 0.96 & \\
\hline & & $F^{2}$ & 0.015 & 33.01 & 28.34 & 100.80 & \\
\hline \multirow[t]{2}{*}{4} & Semi-logarítmico & $R^{2}$ & 0.003 & 0.98 & 0.51 & 0.80 & \\
\hline & & $F^{2}$ & 0.012 & 394.12 & 4.22 & 19.72 & \\
\hline \multirow[t]{2}{*}{5} & $1 / x$ & $R^{2}$ & 0.031 & 0.64 & 0.24 & 0.68 & \\
\hline & & $F^{2}$ & 0.130 & 7.36 & 1.31 & 8.51 & \\
\hline \multirow[t]{2}{*}{6} & $x^{2}$ & $R^{2}$ & 0.053 & 0.40 & 0.86 & 0.95 & \\
\hline & & $F^{2}$ & 0.225 & 2.68 & 26.30 & 93.09 & \\
\hline \multirow[t]{2}{*}{7} & Raíz cuadrada $y$ & $R^{2}$ & 0.017 & 0.98 & 0.81 & 0.97 & \\
\hline & & $F^{2}$ & 0.071 & 210.90 & 17.74 & 149.56 & \\
\hline
\end{tabular}


Cuadro 3. Cálculo del peso fresco del leño útil para la elaboración de carbón de las dos especies de Prosopis estudiadas, con base en los datos obtenidos del muestreo no destructivo y aplicando los modelos de regresión respectivamente seleccionados. Para P. palmeri se usó la cobertura $\left(\mathrm{m}^{2}\right)$ de 1,283 individuos con el fin de estimar la cantidad de leño en pie por unidad de área. Para P. articulata se empleó el perímetro basal del tronco (m) de 755 plantas con el mismo propósito (intervalo de confianza 95\%).

\begin{tabular}{|l|c|c|c|c|c|}
\hline \multirow{2}{*}{\multicolumn{1}{|c|}{ Prosopis palmeri }} & \multicolumn{5}{c|}{ Sitio } \\
\cline { 2 - 6 } & 1 & 2 & 3 & 4 & 5 \\
\hline Número de individuos & 232 & 287 & 259 & 281 & 224 \\
\hline Cobertura sumaria $\left(\mathrm{m}^{2}\right)$ & $1,178.0$ & $1,765.4$ & $1,543.7$ & $1,709.5$ & $1,006.8$ \\
\hline Promedio de cobertura $\left(\mathrm{m}^{2}\right)$ & 5.1 & 6.8 & 5.4 & 6.1 & 4.5 \\
\hline Desviación estándar & 2.1 & 2.3 & 2.3 & 2.5 & 1.7 \\
\hline Coeficiente de variación & 0.4 & 0.3 & 0.4 & 0.4 & 0.4 \\
\hline Peso sumario (kg) & $1,510.5$ & $2,602.7$ & $1,921.4$ & $2,453.3$ & $1,268.6$ \\
\hline Peso promedio x individuo $(\mathrm{kg})$ & 6.5 & 10.0 & 6.7 & 8.7 & 5.7 \\
\hline Desviación estándar & 3.0 & 5.9 & 1.4 & 5.3 & 2.2 \\
\hline Coeficiente de variación & 0.5 & 0.6 & 0.2 & 0.6 & 0.4 \\
\hline
\end{tabular}

\begin{tabular}{|l|c|c|c|c|c|c|}
\hline \multirow{2}{*}{\multicolumn{1}{|c|}{ Prosopis articulata }} & \multicolumn{7}{c|}{ Sitio } \\
\cline { 2 - 7 } & 1 & 2 & 3 & 4 & 5 & 6 \\
\hline Número de individuos & 129 & 130 & 126 & 79 & 178 & 113 \\
\hline Perímetro sumario (m) & 109.2 & 117.5 & 110.0 & 42.4 & 128.5 & 89.7 \\
\hline Promedio del perímetro (m) & 0.8 & 0.9 & 0.9 & 0.6 & 0.7 & 0.8 \\
\hline Desviación estándar & 0.4 & 0.4 & 0.3 & 0.2 & 0.3 & 0.3 \\
\hline Coeficiente de variación & 0.5 & 0.4 & 0.4 & 0.3 & 0.4 & 0.4 \\
\hline Peso sumario (kg) & $53,809.3$ & $52,136.2$ & $42,583.5$ & $7,290.1$ & $35,981.2$ & $30,442.2$ \\
\hline Peso promedio x individuo (kg) & 417.1 & 401.0 & 338.0 & 92.3 & 202.1 & 269.4 \\
\hline Desviación estándar & 464.2 & 365.0 & 288.6 & 75.3 & 169.7 & 234.7 \\
\hline Coeficiente de variación & 1.1 & 0.9 & 0.9 & 0.9 & 0.8 & 0.9 \\
\hline
\end{tabular}


los valores totales y promedio con su desviación estándar de la respectiva variable independiente empleada (cobertura en $P$. palmeri y perímetro basal en $P$. articulata), así como el coeficiente de variación correspondiente (desviación estándar/valor promedio). En un segundo inciso se anota la biomasa del leño útil para cada caso obtenida al sustituir en el modelo respectivo el valor de la variable independiente; se incluye el peso promedio por individuo, su desviación estándar y el coeficiente de variación. Cabe observar la diferencia hasta en un orden de magnitud de tonelaje promedio de $P$. articulata $(37,040 \mathrm{~kg} / \mathrm{ha})$ respecto a $P$. palmeri $(2,045 \mathrm{~kg} / \mathrm{ha})$.

La Fig. 2 ilustra la dispersión de los seis puntos que conforman la recta de regresión obtenida (puntos cerrados), y sobrepuestos (puntos abiertos) los respectivos calculados con el modelo obtenido, que en cada caso son 755 para $P$. articulata (a) y 1,283 para $P$. palmeri (b), ambos se muestran en el contexto del intervalo de confianza de $95 \%(\mathrm{p}<0.05)$. En el eje $x$ se representa el logaritmo natural (ln) del perímetro basal (gráfica a) y la cobertura (gráfica b), en el eje y los valores esperados en logaritmo natural del peso del leño según el modelo empleado. En todos los casos estos puntos se encuentran ubicados dentro de los límites de confianza de la regresión lineal usada.

Los resultados de la determinación del peso específico arrojaron las siguientes cifras: en $P$. palmeri, peso seco $=1.283 \mathrm{gr} / \mathrm{cm}^{3}$, peso fresco $=1.287$ $\mathrm{gr} / \mathrm{cm}^{3}$. Mientras que en $P$. articulata, peso $\mathrm{seco}=1.045 \mathrm{gr} / \mathrm{cm}^{3}$, y en peso fresco $=1.063 \mathrm{gr} / \mathrm{cm}^{3}$. Lo anterior revela una notable diferencia entre las dos especies, pero también señala la circunstancia de que el secado no altera casi en absoluto el peso de la madera.

\section{DISCUSIÓN Y CONCLUSIONES}

Una de las limitaciones del presente estudio es el número relativamente reducido de muestras (seis por especie) a partir del cual se desarrolla el análisis. En teoría, una cantidad más elevada de muestreos permitiría incluir un mayor intervalo de la variación de la relación entre la dimensión del árbol y su peso, y así obtener resultados con un nivel de confianza más estrecho. Cabe señalar que por razones éticas y de limitaciones de infraestructura para trozar más árboles se consideró que el número de derribos fuera el anteriormente anotado. Con el propósito de compensar esta aparente limitación, los autores eligieron árboles completos, aún sin evidencias de cortes por leñadores, y que parecieron ser también los de mayores dimensiones observados en los diferentes recorridos realizados en el área. 


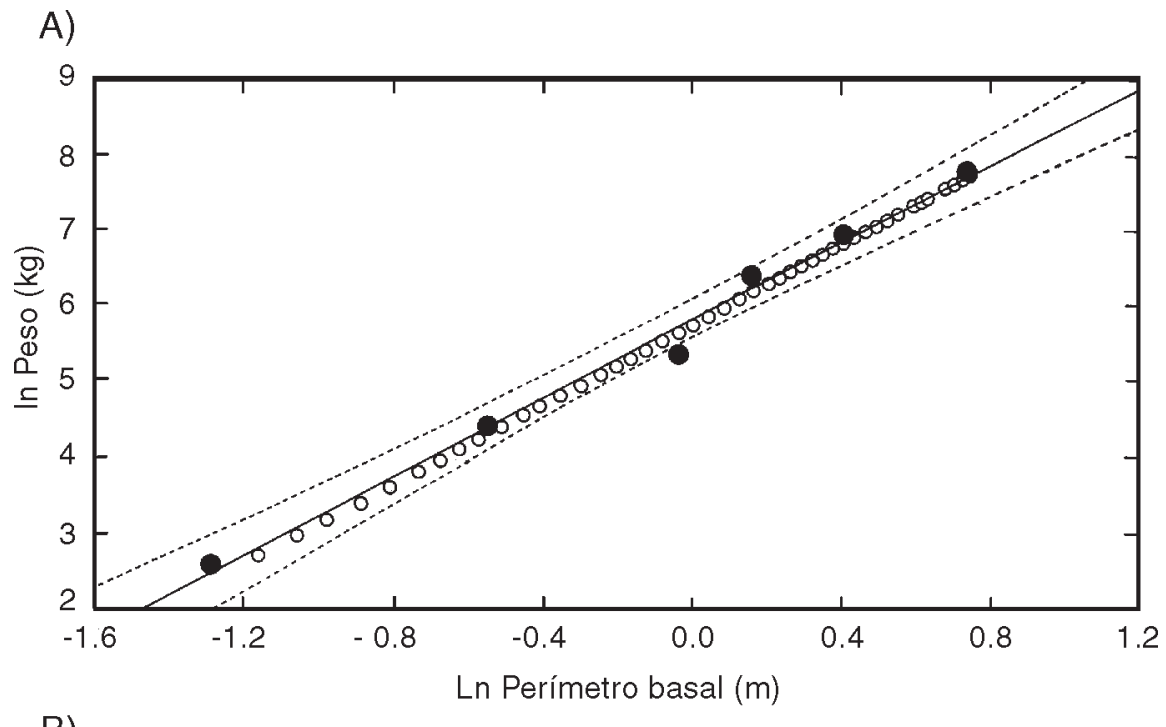

B)

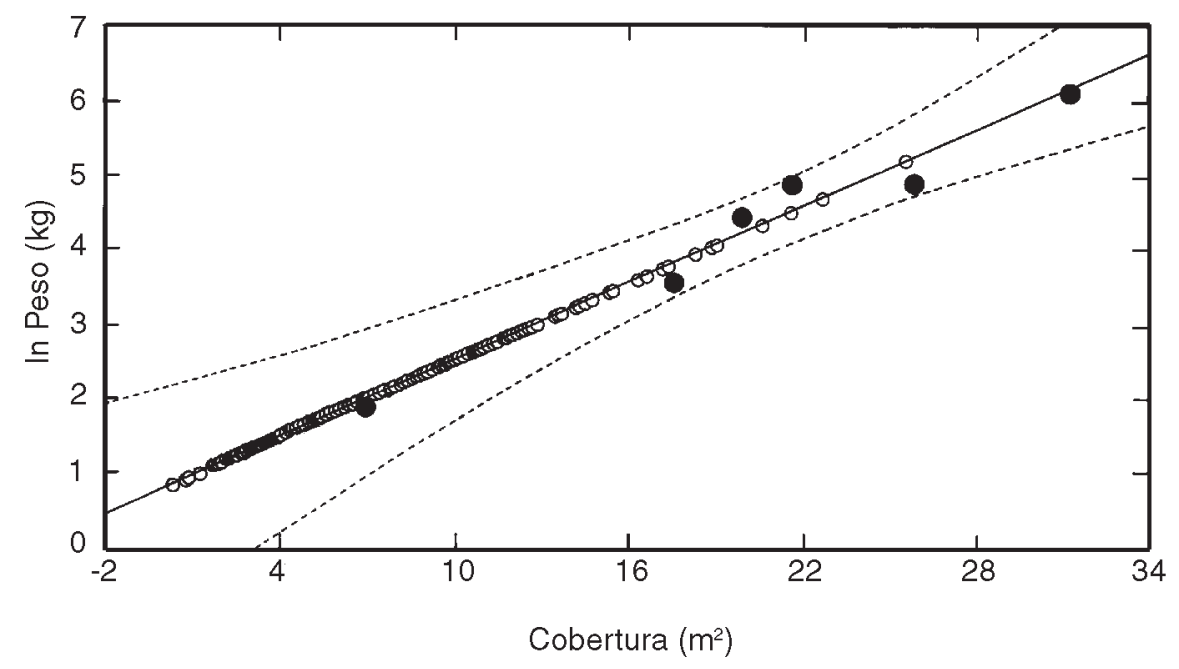

Fig. 2. Ilustración de la distribución de los datos obtenidos de los muestreos y de los calculados con base en los modelos de regresión respectivos dentro de un nivel de confianza de $95 \%$ $(p<0.05)$. Los puntos cerrados corresponden a las 6 determinaciones que dieron lugar al modelo para cada especie. Los puntos abiertos indican las estimaciones del peso del leño con base en muestreos no destructivos usando la variable dependiente $(x)$ respectiva. A) Prosopis articulata, con datos de ambos ejes expresados en su logaritmo natural (ln) en vista del modelo logarítmico utilizado $[\ln (y)=\mathrm{b} \ln (x)+\ln (\mathrm{a})]$. B) Prosopis palmeri, con la transformación a la escala logarítmica sólo en el eje de la variable independiente $(y)$, dado el uso del modelo exponencial $[\ln (y)=\mathrm{b} x+\mathrm{a}]$. 
Los resultados obtenidos muestran que no necesariamente la misma medida morfométrica, expresada como la variable independiente de un modelo, puede ser de utilidad en dos o más especies de Prosopis cuando es empleada para inferir el peso del leño. La razón puede encontrarse al considerar que a pesar de que se trata de plantas arbóreas, cada una muestra un particular patrón de crecimiento. En el caso del "mezquite amargo" $P$. articulata, se trata de un árbol cuyos individuos suelen superar los $5 \mathrm{~m}$ de altura, con un crecimiento comparativamente más rápido que la otra especie, su gran perímetro basal es el efecto del crecimiento secundario del leño que le permite soportar varios cientos de kilogramos de peso total que logran alcanzar por lo general los individuos en el lapso de unos 100 años, de acuerdo con informantes rurales.

En contraste, el "palo fierro", P. palmeri, desarrolla comúnmente plantas de 2 a $3 \mathrm{~m}$, y excepcionalmente $5 \mathrm{~m}$ de alto, por lo que el papel de la resistencia mecánica del tronco no parece tener la misma importancia que en el primer caso, ya que se trata de organismos de lento crecimiento. En consecuencia, otra variable estructural, como el crecimiento horizontal del ramaje, determinado como su cobertura, guarda mejor relación con su biomasa leñosa. Excepcionalmente se encuentran ejemplares que sobrepasan los $5 \mathrm{~m}$ de altura, pero nunca se presentan como individuos frondosos, ya que el daño durante los siglos de edad se refleja en su crecimiento.

Los resultados de los muestreos extensivos no destructivos indican que varios individuos de $P$. articulata, de los 755 inventariados, se acercaron al límite superior de $2 \mathrm{~m}$ de perímetro basal (ver registros selectivos que dieron lugar al modelo en el Cuadro 1), lo cual indica que tal medida marca el límite superior de crecimiento para los añejos individuos de la especie en esta región geográfica. El resto de las mediciones se distribuyen con cierta uniformidad dentro del intervalo de datos del modelo del Cuadro 1, como puede observarse al compararlo con la Fig. 2. Por el contrario, en el muestreo extensivo de 1283 individuos de $P$. palmeri, si bien sólo unas pocas mediciones se encuentran próximas al límite superior de la cobertura, la mayor parte de tales registros extensivos de esta variable se agregaron hacia la mitad inferior del conjunto de los registros selectivos (comparar Cuadro 1 y Fig. 2), lo que indica que las tallas grandes son excepcionales, producto de una particular estructura poblacional, o bien que el actual aprovechamiento refleja el resultado de un proceso de extracción selectivo sobre árboles de tamaños grandes.

Aunque formalmente no se presentan resultados que indiquen la estructura poblacional por edades, o algún otro parámetro que permita inferirla, la secuencia de los cientos de registros calculados que se muestran en la Fig. 2 señala la tendencia de la agregación de los individuos de cada especie en función de la variable correspondiente, que con ciertas reservas sugerirían "clases de edad". Debido a la extracción selectiva de las tallas grandes, tal tendencia subestima a las tallas 
mayores. Esta afirmación es particularmente cierta en el caso del palo fierro, $P$. palmeri, ya que por el grado de dureza su leño ha sido tradicionalmente preferido por los rancheros locales para múltiples usos.

El mezquite amargo, $P$. articulata, si bien presenta una mayor densidad de biomasa por unidad de superficie que la otra especie, cabe resaltar que su hábitat (los cauces de arroyos) cubre proporcionalmente una menor área que la correspondiente al del palo fierro. Una estimación comparativa gruesa del terreno ocupado por el hábitat de cada especie (laderas y mesas vs. arroyos), basada en el análisis de un mapa de la zona, y con ayuda del trazo de polígonos analizados por un sistema de información geográfica (SIG), muestra que menos de $1.0 \%$ de la superficie es ocupada por los cauces de arroyos (el hábitat de P. articulata) en una extensión de $723 \mathrm{~km}^{2}$ de la cuenca del sistema de arroyos Tepentú-La Picota, dentro de las cotas altitudinales de 100 y $400 \mathrm{~m}$.

Considerando los 49,900 $\mathrm{m}^{3}$ de madera en rollo que oficialmente se declararon aprovechados, de acuerdo con los permisos del período 1990-1997 en los municipios de La Paz y Comondú (Anónimo, 1998), este volumen representaría en peso la extracción de 6,635 a 8,033 ton de leño en promedio anual, aplicando la conversión en función de los valores de peso específico antes citados y en la inteligencia de que los valores extremos corresponden a la condición teórica de uso exclusivo de una u otra especie. Si se tratase de aprovechamientos intensivos, que afectaran uniformemente sólo la superficie requerida para obtener ese volumen, y si se compara esta cantidad con la biomasa de leño aprovechable de cada especie por unidad de superficie, señalada en el Cuadro 3, resultaría que: a) de provenir exclusivamente de mezquite amargo, la extracción anual equivaldría al aprovechamiento de individuos de tallas propias para el propósito en una superficie de 179.1 ha en los bordes de arroyos; b) de tratarse exclusivamente de palo fierro, equivaldría al aprovechamiento anual de individuos en 3,928 ha en mesas y lomeríos.

Afortunadamente, la extracción ocurre en una amplia superficie, afectando individuos de un sitio y de otro, y aprovechando en principio "leñas muertas", lo que permite amortiguar el impacto sobre los procesos de crecimiento y regeneración natural de cada población. Sin embargo, algunos factores se suman para acentuar el impacto, como es el uso ancestral del recurso para energía y postería, al igual que la extracción clandestina, de la cual no existen datos ni estimaciones. De manera opuesta, bajo cierta perspectiva la actividad ganadera puede considerarse un factor que favorece las etapas de dispersión y germinación de semillas, y resulta también positivo el hecho de que algunos tocones tienen la capacidad de rebrotar después de cortados.

De acuerdo con las cifras oficiales (Anónimo, 1998), el beneficio económico recibido por la extracción, elaboración, transporte y venta de carbón a través de 
8 años puede alcanzar el equivalente a $\$ 1,300,000.00$ dólares, cantidad que si se repartiera equitativamente entre las decenas de familias que se ocupan de esta actividad (80-120), bien podría alcanzar para proporcionar niveles de ingreso de una familia promedio urbana. Sin embargo, una encuesta privada reciente (Sociedad Niparajá, 2003, com. pers.) señala que los beneficios económicos se encuentran sesgados hacia los compradores mayoristas, quienes adquieren alrededor de $50 \%$ de la producción. Estos últimos compran el kilogramo del producto en la carbonera a $\$ 1.90$ pesos o en la Cd. de La Paz hasta en $\$ 4.00$ pesos. La reventa del carbón al menudeo se ubica entre $\$ 7.00$ y $\$ 7.50$ pesos por kilo. La otra mitad es adquirida directamente por cadenas de restaurantes que lo usan para cocinar carne de res y pollo al estilo peninsular.

Son varios los aspectos que deberán abordarse en las siguientes etapas de esta investigación, tendientes a diseñar un plan de manejo silvícola para el recurso vegetal en cuestión. Uno de ellos es el relacionado con la productividad de cada especie, para lo cual es indispensable contar con información sobre la edad de cada individuo, relacionándola estadísticamente con variables como el peso del leño; lo anterior no parece ser una empresa fácil, ya que en los mezquites los anillos de crecimiento no son estrictamente anuales (Flinn et al., 1994). Otro más es el relativo a la tasa de reclutamiento en las clases de edades o de tamaños de las poblaciones, datos que permitirían establecer una tabla de vida para cada especie. Finalmente, sería necesario inventariar a una escala mayor las existencias de estos recursos a través de sistemas de información geográfica (SIG).

\section{AGRADECIMIENTOS}

Deseamos reconocer la valiosa participación de Franco Cota y Miguel Domínguez en el duro trabajo de campo; de Jesús Quiñones, del Instituto Nacional de Ecología de Baja California Sur, quien nos facilitó información de archivos; de Don Manuel Gómez Higuera, propietario del rancho "La Poza Sola", quien amablemente nos brindó facilidades en su propiedad, así como de sus hijos Álvaro y Antonio. Agradecemos a la Sociedad de Historia Natural Niparajá A. C. de La Paz, B. C. S. la valiosa información del estudio de mercado de productos pesqueros, agropecuarios y rurales. Rocío Coria elaboró el mapa de ubicación.

\section{LITERATURA CITADA}

Anónimo. 1981a. Carta de uso del suelo y vegetación 1:1,000,000. Hoja La Paz. Secretaría de Programación y Presupuesto, Dirección General de Geografía del Territorio Nacional. México D.F. 
Anónimo. 1981b. Carta de climas 1:1,000,000. Hoja La Paz. Secretaría de Programación y Presupuesto, Dirección General de Geografía del Territorio Nacional. México D.F.

Anónimo. 1996. Estudio hidrológico del Estado de Baja California Sur. Instituto Nacional de Estadística e Información Geográfica (INEGI)-Gobierno del Estado de Baja California Sur. Aguascalientes, Ags. 206 pp.

Anónimo. 1998. Estadística de autorizaciones y evolución del volumen y valor de la producción forestal (1990-1997) municipios de La Paz y Comondú. Secretaría del Medio Ambiente, Recursos Naturales y Pesca (SEMARNAP) Baja California Sur. La Paz, Baja California Sur. 9 pp.

Carrillo-García, A., J. L. León de la Luz, Y. Bashan y G. J. Bethlenfalvay. 1999. Nurse plants, mycorrhizae, and plant establishment in a disturbed area of the Sonoran Desert. Restoration Ecology 7(4): 321-335.

Flinn, R. C., S. Archer, T. W. Boutton y T. Harlan. 1994. Identification of annual rings in arid-land woody plants: Prosopis glandulosa. Ecology 75(3): 850-853.

Kvalseth, T. O. 1985. Cautionary note about $R^{2}$. American Statistics 39: 279-285.

Murray, R. B. y M. Q. Jacobson. 1982. An evaluation of dimension analysis for predicting shrub biomass. Journal of Range Management 35: 451-454.

Osuna Leal, E. 1989. Comparación de la eficiencia de tres métodos para la elaboración de carbón con mezquite (Prosopis spp.). Tesis de Licenciatura (Ingeniero Agrónomo). Universidad Autónoma de Baja California Sur, La Paz, Baja California Sur. 67 pp.

Patón, D., P. Azócar y J. Tovar. 1998. Growth and productivity in forage biomass in relation to the age assessed by dendrochronology in the evergreen shrub Cistus ladanifer (L.) using different regression models. Journal of Arid Environments 38: 221-235.

Rzedowski, J. 1978. Vegetación de México. Editorial Limusa. México, D.F. 431 pp.

Shreve, F. e I. L. Wiggins. 1964. Vegetation and flora of the Sonoran Desert. Stanford University Press. Stanford, CA. 1740 pp.

Turner, M. R., J. W. Bowers y T. L. Burgess. 1995. Sonoran desert plants: an ecological atlas. The University of Arizona Press. Tucson, AZ. 504 pp.

Vandermeer, J. 1980. Saguaros and nurse trees: a new hypothesis to account for population fluctuations. Southwestern Naturalist 25: 357-360.

Vora, R. S. 1988. Predicting biomass of five shrub species in northeastern California. Journal of Range Management 41(1): 63-65.

Wiggins, I. L. 1980. Flora of Baja California. Stanford University Press. Stanford, CA.1025 pp.

Zar, J. H. 1984. Biostatistical analysis. Prentice Hall, Englewood Cliffs, N.J. 718 pp. 